\title{
LINKEDNESS AND ORDERED CYCLES IN DIGRAPHS
}

\author{
DANIELA KÜHN AND DERYK OSTHUS
}

\begin{abstract}
Given a digraph $D$, let $\delta(D):=\min \left\{\delta^{+}(D), \delta^{-}(D)\right\}$ be the minimum degree of $D$. We show that every sufficiently large digraph $D$ with $\delta(D) \geq n / 2+\ell-1$ is $\ell$-linked. The bound on the minimum degree is best possible and confirms a conjecture of Manoussakis [16. We also determine the smallest minimum degree which ensures that a sufficiently large digraph $D$ is $k$-ordered, i.e. that for every sequence $s_{1}, \ldots, s_{k}$ of distinct vertices of $D$ there is a directed cycle which encounters $s_{1}, \ldots, s_{k}$ in this order.
\end{abstract}

\section{INTRODUCTION}

The minimum degree $\delta(D)$ of a digraph $D$ is the minimum of its minimum outdegree $\delta^{+}(D)$ and its minimum indegree $\delta^{-}(D)$. When referring to paths and cycles in digraphs we always mean that these are directed without mentioning this explicitly. A digraph $D$ is $\ell$-linked if $|D| \geq 2 \ell$ and if for every sequence $x_{1}, \ldots, x_{\ell}, y_{1}, \ldots, y_{\ell}$ of distinct vertices there are disjoint paths $P_{1}, \ldots, P_{\ell}$ in $D$ such that $P_{i}$ joins $x_{i}$ to $y_{i}$. Since this is a very strong and useful property to have in a digraph, the question of course arises how it can be forced by other properties.

In the case of (undirected) graphs, much progress has been made in this direction. In particular, linkedness is closely related to connectivity: Bollobás and Thomason [2] showed that every $22 k$-connected graph is $k$-linked (this was recently improved to $10 k$ by Thomas and Wollan [17]). However, for digraphs the situation is quite different: Thomassen [18] showed that for all $k$ there are strongly $k$-connected digraphs which are not even 2-linked.

Our first result determines the minimum degree forcing a (large) digraph to be $\ell$-linked, which confirms a conjecture of Manoussakis [16] for large digraphs.

Theorem 1. Let $\ell \geq 2$. Every digraph $D$ of order $n \geq 1600 \ell^{3}$ which satisfies $\delta(D) \geq n / 2+\ell-1$ is $\ell$-linked.

It is not hard to see that the bound on minimum degree in Theorem 1 is best possible (see Proposition 3). It is also easy to see that for $\ell=1$ the correct bound is $\delta(D) \geq\lfloor n / 2\rfloor$. The cases $\ell=2,3$ of Theorem 1 were proved by Heydemann and Sotteau [9] and Manoussakis [16] respectively. Manoussakis [16] also determined the number of edges which force a digraph to be $\ell$-linked. A discussion of these and related results can be found in the monograph by Bang-Jensen and Gutin [1].

Note that it does not make sense to ask for the minimum outdegree of a digraph $D$ which ensures that $D$ is $\ell$-linked (or similarly, to ask for the minimum indegree). Indeed, the digraph obtained from a complete digraph $A$ of order $n-1$ by adding a new vertex $x$ which sends an edge to every vertex in $A$ has minimum outdegree $n-2$ but is not even 1-linked.

A slightly weaker notion is that of a $k$-ordered digraph: a digraph $D$ is $k$-ordered if $|D| \geq k$ and if for every sequence $s_{1}, \ldots, s_{k}$ of distinct vertices of $D$ there is a cycle 
which encounters $s_{1}, \ldots, s_{k}$ in this order. It is not hard to see that every $\ell$-linked digraph is also $\ell$-ordered. Conversely, every $2 \ell$-ordered digraph $D$ is also $\ell$-linked: if $x_{1}, \ldots, x_{\ell}, y_{1}, \ldots, y_{\ell}$ is a sequence of vertices as in the definition of $\ell$-linkedness then a cycle which encounters $x_{1}, y_{1}, x_{2}, y_{2}, \ldots, x_{\ell}, y_{\ell}$ in this order would yield the paths required for the linking. The next result says that as far as the minimum degree is concerned it is no harder to guarantee the $2 \ell$ paths forming such a cycle than to guarantee just the $\ell$ paths required for the linking. In particular, note that Theorem 2 immediately implies Theorem 1 .

Theorem 2. Let $k \geq 2$. Every digraph $D$ of order $n \geq 200 k^{3}$ which satisfies $\delta(D) \geq(n+k) / 2-1$ is k-ordered.

Again, the bound on the minimum degree is best possible (see Proposition 4). Moreover, it is easy to see that if $k=1$ then the correct bound is $\delta(D) \geq n / 2-1$. The proof of Theorem 2 yields paths between the $k$ 'special' vertices whose length is at most 6 and it is also easy to translate the proof into an algorithm which finds these paths in polynomial time (see the remarks after the end of the proof).

Somewhat surprisingly, the minimum degree in both theorems is not quite the same as in the undirected case: Kawarabayashi, Kostochka and Yu [12] proved that the smallest minimum degree which guarantees a graph on $n$ vertices to be $\ell$-linked is $\lfloor n / 2\rfloor+\ell-1$ for large $n$. (Egawa et al. [4] independently determined the smallest minimum degree which guarantees the existence of $\ell$ disjoint cycles containing $\ell$ specified independent edges, which is clearly a very similar property.) Kierstead, Sarközy and Selkow [13] proved that the smallest minimum degree which guarantees a graph on $n$ vertices to be $k$-ordered is $\delta(D) \geq\lceil n / 2\rceil+\lfloor k / 2\rfloor-1$ for large $n$. So in the undirected case the ' $2 \ell$-ordered' result does not quite imply the ' $\ell$-linked' result. The proofs in [4, 12, 13] do not seem to generalize to digraphs.

\section{FURTHER WORK AND OPEN PROBLEMS}

In a sequel to this paper, we hope to apply Theorem 2 to obtain the following stronger results, which would also generalize the theorem of Ghouila-Houri [6] that any digraph $D$ on $n$ vertices with $\delta(D) \geq n / 2$ contains a Hamilton cycle: we aim to apply Theorem 2 to show that if $k \geq 2$ and $D$ is a sufficiently large digraph whose minimum degree is as in Theorem 2 then $D$ is even $k$-ordered Hamiltonian, i.e. for every sequence $s_{1}, \ldots, s_{k}$ of distinct vertices of $D$ there is a Hamilton cycle which encounters $s_{1}, \ldots, s_{k}$ in this order. One can use this to prove that the minimum degree condition in Theorem 1 already implies that the digraph $D$ is Hamiltonian $\ell$-linked, i.e. the paths linking the pairs of vertices span the entire vertex set of $D$. Note that this in turn would immediately imply that $D$ is $\ell$-arc ordered Hamiltonian, i.e. $D$ has a Hamilton cycle which contains any $\ell$ disjoint edges in a given order. Note that in each case the examples in Section 3 show that the minimum degree condition would be best possible. Undirected versions of these statements were first obtained by Kierstead, Sarközy and Selkow [13] and Egawa et al. 4] respectively (and a common generalization of these in [3]).

For graphs, the concepts ' $\ell$-linked' and ' $k$-ordered' were generalized to ' $H$-linked' by Jung [11]: a graph $G$ is $H$-linked if $G$ contains a subdivision of $H$ with prescribed branch vertices (so $G$ is $k$-ordered if and only if it is $C_{k}$-linked). The minimum 
degree which forces a graph to be $H$-linked for an arbitrary $H$ was determined in [5, 14, 15, 7]. Clearly, one can ask similar questions also for digraphs.

Finally, we believe that the bound on $n$ which we require in Theorem 2 (and thus in Theorem 1) can be reduced to one which is linear in $k$.

\section{NotATiOn AND EXTREMAL EXAMPLES}

Before we discuss the examples showing that the bounds on the minimum degree in Theorems 1 and 2 are best possible, we will introduce the basic notation used throughout the paper. A digraph $D$ is complete if every pair of vertices of $D$ is joined by edges in both directions. The order $|D|$ of a digraph $D$ is the number of its vertices. We write $N^{+}(x)$ for the outneighbourhood of a vertex $x$ and $d^{+}(x):=$ $\left|N^{+}(x)\right|$ for its outdegree. Similarly, we write $N^{-}(x)$ for the inneighbourhood of a vertex $x$ and $d^{-}(x):=\left|N^{-}(x)\right|$ for its indegree. We set $d(x):=\min \left\{d^{+}(x), d^{-}(x)\right\}$. Given a set $A$ of vertices of $D$, we write $N_{A}^{+}(x)$ for the set of all outneighbours of $x$ in $A . N_{A}^{-}(x), d_{A}^{+}(x)$ and $d_{A}^{-}(x)$ are defined similarly. Given two vertices $x, y$ of a digraph $D$, an $x-y$ path in $D$ is a directed path which joins $x$ to $y$. Given two disjoint vertex sets $A$ and $B$ of $D$, an $A$-B edge is an edge $\overrightarrow{a b}$ where $a \in A$ and $b \in B$.

The following proposition shows that the bound on the minimum degree in Theorem 1 cannot be reduced.

Proposition 3. For every $\ell \geq 2$ and every $n \geq 2 \ell$ there exists a digraph $D$ on $n$ vertices with minimum degree $\lceil n / 2\rceil+\ell-2$ which is not $\ell$-linked.

Proof. We will distinguish the following cases.

Case 1. $n$ is even.

Let $D$ be the digraph which consists of complete digraphs $A$ and $B$ of order $n / 2+$ $\ell-1$ which have precisely $2 \ell-2$ vertices in common. To see that $D$ is not $\ell$ linked let $x_{1}, \ldots, x_{\ell-1}, y_{1}, \ldots, y_{\ell-1}$ denote the vertices in $A \cap B$. Pick some vertex $x_{\ell} \in A \backslash B$ and some vertex $y_{\ell} \in B \backslash A$. Then $D$ does not contain disjoint paths between $x_{i}$ and $y_{i}$ for all $i=1, \ldots, \ell$. The minimum degree of $D$ is attained by the vertices in $(A \backslash B) \cup(B \backslash A)$ and thus is as desired.

Case 2. $n$ is odd.

In this case, we define $D$ as follows. Let $A$ and $B$ be disjoint complete digraphs of order $\lceil n / 2\rceil-\ell-1$. Add a complete digraph $X$ of order $2 \ell-3$ and join all vertices in $X$ to all vertices in $A \cup B$ with edges in both directions. Add a set $S:=\left\{x_{1}, x_{2}, y_{1}, y_{2}\right\}$ of 4 new vertices such that each vertex in $S$ is joined to each vertex in $X$ with edges in both directions. Moreover, we add all the edges between different vertices in $S$ except for $\overrightarrow{x_{1} y_{1}}$ and $\overrightarrow{x_{2} y_{2}}$. Finally, we connect the vertices in $S$ to the vertices in $A \cup B$ as follows. Both $x_{1}$ and $y_{1}$ receive edges from every vertex in $B$ and send edges to every vertex in $A$. Additionally, $x_{1}$ will receive an edge from every vertex in $A$ and $y_{1}$ will send an edge to every vertex in $B$. Both $x_{2}$ and $y_{2}$ receive edges from every vertex in $A$ and send edges to every vertex in $B$. Additionally, $x_{2}$ will receive an edge from every vertex in $B$ and $y_{2}$ will send an edge to every vertex in $A$ (see Figure 1).

To check that $D$ has the required minimum degree, consider first any vertex $a \in$ $A$. As $a$ sends edges to 3 vertices in $S$ and receives edges from 3 such vertices, 


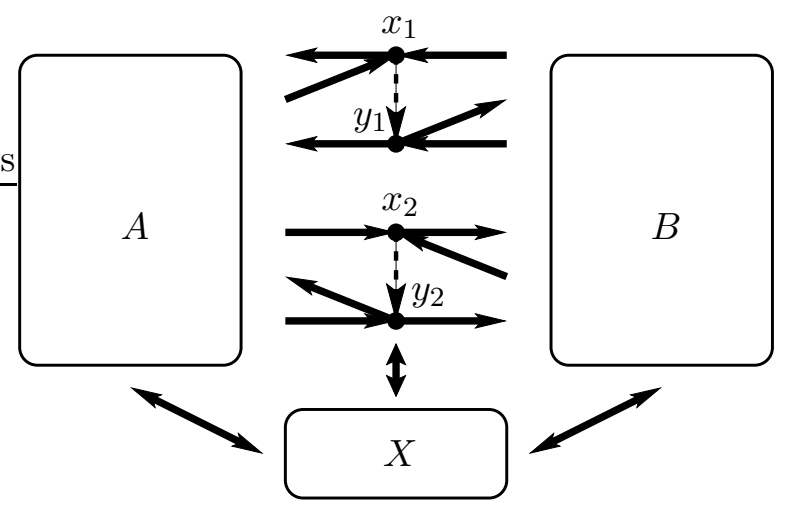

Figure 1. The digraph $D$ in Case 2 of Proposition 3 , The dashed arrows indicate the missing edges between $x_{1}$ and $y_{1}$ and between $x_{2}$ and $y_{2}$.

we have that $d(a)=|A|-1+|X|+3=\lceil n / 2\rceil+\ell-2$. It follows similarly that the vertices in $B$ have the correct degree. Thus consider any vertex $s \in S$. Then $s$ sends edges to all vertices in $A$ or to all vertices in $B$ (or both) and $s$ receives edges from all vertices in $A$ or from all vertices in $B$ (or both). Thus $d(s)=|A|+|X|+2=\lceil n / 2\rceil+\ell-2$. It is easy to check that the vertices in $X$ have the required degree and thus $\delta(D)=\lceil n / 2\rceil+\ell-2$.

To see that $D$ is not $\ell$-linked, let $x, x_{3}, \ldots, x_{\ell}, y_{3}, \ldots, y_{\ell}$ denote the vertices in $X$. Then we cannot link $x_{i}$ to $y_{i}$ for each $i=1, \ldots, \ell$ since every $x_{1}-y_{1}$ path must meet $X \cup\left\{x_{2}, y_{2}\right\}$ (and thus would contain $x$ ) and the analogue is true for every $x_{2}-y_{2}$ path.

We conclude this section with the examples showing that the bound on the minimum degree in Theorem 2 is best possible.

Proposition 4. For every $k \geq 2$ and every $n \geq 2 k$ there exists a digraph $D$ on $n$ vertices with minimum degree $\lceil(n+k) / 2\rceil-2$ which is not $k$-ordered.

Proof. We will distinguish the following cases.

Case 1. $k \geq 3$ is odd and $n$ is even.

In this case, we define $D$ as follows. Let $A$ and $B$ be disjoint complete digraphs of order $n / 2-k+1$. Add a complete digraph $X$ of order $k-2$ and join all its vertices to all vertices in $A \cup B$ with edges in both directions. Add new vertices $s_{1}, \ldots, s_{k}$ such that every $s_{i}$ is joined to all vertices in $X$ with edges in both directions. Moreover, we add all the edges $\overrightarrow{s_{i} s_{j}}$ for $j \neq i, i+1$ where $s_{k+1}:=s_{1}$. We also add the edge $\overrightarrow{s_{1} s_{2}}$. Finally, we connect the $s_{i}$ to the vertices in $A \cup B$ as follows. Both $s_{1}$ and $s_{2}$ receive edges from every vertex in $B$ and send edges to every vertex in $B$. Additionally, $s_{1}$ will send an edge to every vertex in $A$ and $s_{2}$ will receive an edge from every vertex in $A$. Each of $s_{3}, s_{5}, \ldots, s_{k}$ receives an edge from every vertex in $A$ and sends an edge to every vertex in $A$. Each of $s_{4}, s_{6}, \ldots, s_{k-1}$ receives an edge from every vertex in $B$ and sends an edge to every vertex in $B$ (see Figure 2). Let us now check that the minimum degree of the digraph $D$ thus obtained is as required. Let $S:=\left\{s_{1}, \ldots, s_{k}\right\}$. Note that each 


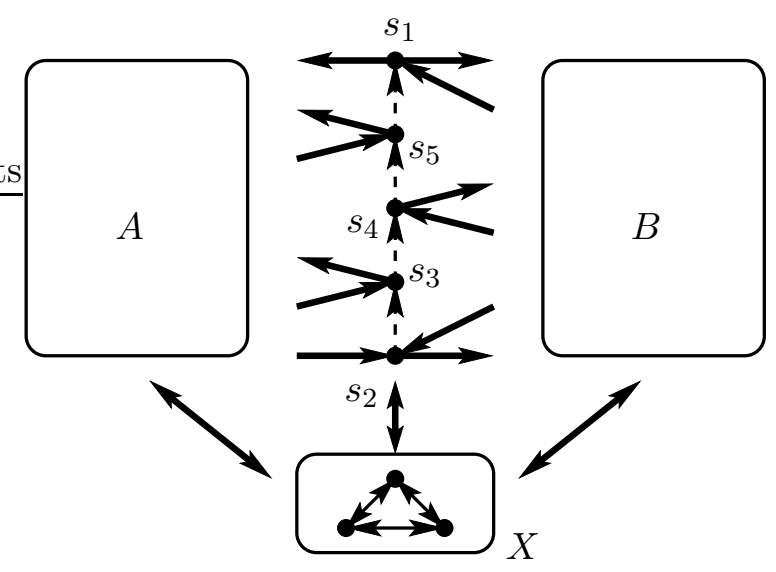

Figure 2. The digraph $D$ for $k=5$ in Case 1 of Proposition 4 , The dashed arrows indicate missing edges between the vertices $s_{i}$.

vertex $v \in A \cup B$ sends edges to precisely $(k+1) / 2$ vertices in $S$ and receives edges from precisely that many vertices. Since $|A|=|B|$, it follows that $d(v)=$ $|A|-1+|X|+(k+1) / 2=n / 2-2+(k+1) / 2=\lceil(n+k) / 2\rceil-2$. Now consider any $s_{i} \in S$. Then $s_{i}$ receives edges from either all vertices in $A$ or all vertices in $B$ (or both) and $s_{i}$ sends edges to either all vertices in $A$ or all vertices in $B$ (or both). Hence $d\left(s_{i}\right) \geq|A|+|X|+|S|-2=n / 2-1+k-2 \geq\lceil(n+k) / 2\rceil-2$. It is easy to check that the degree of the vertices in $X$ is $>\lceil(n+k) / 2\rceil-2$.

To see that $D$ is not $k$-ordered note that every cycle in $D$ which encounters $s_{1}, \ldots, s_{k}$ in this order would use at least one vertex from $X$ between $s_{i}$ and $s_{i+1}$ for every $i \neq 1$ (see Figure 2). But since $|X|=k-2$ this is impossible.

Case 2. $k$ is even.

Let $D$ be the digraph which consists of a complete digraph $A$ of order $\lceil n / 2\rceil+k / 2-1$ and a complete digraph $B$ of order $\lfloor n / 2\rfloor+k / 2$ which has precisely $k-1$ vertices in common with $A$. It is easy to check that $\delta(D)=|A|-1=\lceil(n+k) / 2\rceil-2$. To see that $D$ is not $k$-ordered, pick vertices $s_{1}, s_{3}, \ldots, s_{k-1}$ in $A \backslash B$ and $s_{2}, s_{4}, \ldots, s_{k}$ in $B \backslash A$. Then every cycle in $D$ which encounters $s_{1}, \ldots, s_{k}$ in this order would meet $A \cap B$ when going from $s_{i}$ to $s_{i+1}$, i.e. it would meet $A \cap B k$ times, which is impossible.

Case 3. $k \geq 3$ is odd and $n$ is odd.

This time we take $D$ to be the digraph which consists of two complete digraphs $A$ and $B$ of order $(n+k) / 2-1$ having $k-2$ vertices in common. Then $\delta(D)=$ $|A|-1=(n+k) / 2-2$. To see that $D$ is not $k$-ordered, pick vertices $s_{1}, s_{3}, \ldots, s_{k}$ in $A \backslash B$ and $s_{2}, s_{4}, \ldots, s_{k-1}$ in $B \backslash A$.

Note that in the proof of Proposition 4 we could have omitted the (easy) case when $k$ is even as Proposition 3 already gives a digraph of the required minimum degree which is not $k / 2$-linked and thus not $k$-ordered. 


\section{Proof of Theorem 2}

We first prove Theorem 2 for the case when $k=2$. So suppose that $D$ is a digraph of minimum degree at least $\lceil n / 2\rceil$. Let $s_{1}$ and $s_{2}$ be the vertices which our cycle has to encounter. If $\overrightarrow{s_{1} s_{2}}$ is not an edge then $s_{1}, s_{2} \notin N^{+}\left(s_{1}\right) \cup N^{-}\left(s_{2}\right)$ and so $\left|N^{+}\left(s_{1}\right) \cap N^{-}\left(s_{2}\right)\right| \geq 2 \delta(D)-(n-2) \geq 2$. Similarly, if $\overrightarrow{s_{2} s_{1}}$ is not an edge then $\left|N^{-}\left(s_{1}\right) \cap N^{+}\left(s_{2}\right)\right| \geq 2$. Altogether this shows that there is a cycle of length at most 4 which contains both $s_{1}$ and $s_{2}$.

Thus we may assume that $k \geq 3$ and that $D$ is a digraph of minimum degree at least $\lceil(n+k) / 2\rceil-1$. Let $S:=\left(s_{1}, \ldots, s_{k}\right)$ be the given sequence of vertices of $D$ which our cycle has to encounter. We will call these vertices special and will sometimes also use $S$ for the set of these vertices. We set $s_{k+1}:=s_{1}$. Given a set $I \subseteq[k]$ and a family $T:=\left(t_{i}\right)_{i \in I}$ of positive integers, an $(S, I, T)$-system is a family $\left(\mathcal{P}_{i}\right)_{i \in I}$ where each $\mathcal{P}_{i}$ is a set of $t_{i}$ paths joining $s_{i}$ to $s_{i+1}$ and each path in $\mathcal{P}_{i}$ has length at most 6 and is internally disjoint from $S$, from all other paths in $\mathcal{P}_{i}$ and from the paths in all the other $\mathcal{P}_{j}$. An $(S, I)$-system is an $(S, I, T)$-system where $t_{i}=1$ for all $i \in I$. Thus to prove Theorem 2 we have to show that there exists an $(S,[k])$-system.

Let $I$ be the set of all those indices $i \in[k]$ for which $D$ does not contain at least $6 k$ internally disjoint $s_{i}-s_{i+1}$ paths of length at most 6 .

Claim 1. It suffices to show that $D$ contains an $(S, I)$-system.

Indeed, suppose that $\left(\mathcal{P}_{i}\right)_{i \in I}$ is an $(S, I)$-system in $D$. So each $\mathcal{P}_{i}$ contains precisely one path $P_{i}$. We will show that for every $i \in[k] \backslash I$ we can find an $s_{i} s_{i+1}$ path $P_{i}$ of length at most 6 which meets $S$ only in $s_{i}$ and $s_{i+1}$ such that all the paths $P_{1}, \ldots, P_{k}$ are internally disjoint. We will choose such a path $P_{i}$ for every $i \in[k] \backslash I$ in turn. Suppose that next we want to find $P_{j}$. Recall that since $j \in[k] \backslash I$ the digraph $D$ contains a set $\mathcal{P}$ of at least $6 k$ internally disjoint $s_{j}-s_{j+1}$ paths of length at most 6 . Since at most $5(k-1)+k<6 k$ vertices of $D$ lie in $S$ or in the interior of some of the other paths $P_{i}$, one of the paths in $\mathcal{P}$ must be internally disjoint from $S$ and all the other paths $P_{i}$, and so we can take this path to be $P_{j}$. This proves Claim 1 .

In order to prove the existence of an $(S, I)$-system, choose an $(S, J, T)$-system $\left(\mathcal{P}_{j}\right)_{j \in J}$ in $D$ such that $J \subseteq I$ is as large as possible and subject to this $\sum_{j \in J} t_{j}$ is maximal. Note that $t_{j}<6 k$ for all $j \in J$ since $J \subseteq I$. Assume that $|J|<|I|$. By relabelling the special vertices, we may assume that $k \in I \backslash J$. So we would like to extend $\left(\mathcal{P}_{j}\right)_{j \in J}$ by a suitable $s_{k}-s_{1}$ path. Let $X^{\prime}$ be the set of all those vertices which lie in the interior of some path belonging to $\left(\mathcal{P}_{j}\right)_{j \in J}$. Note that

$$
\left|S \cup X^{\prime}\right|<6 k \cdot 5(k-1)+|S|<30 k^{2}=: k_{0} .
$$

Let $A:=N^{+}\left(s_{k}\right) \backslash\left(S \cup X^{\prime}\right)$ and $B:=N^{-}\left(s_{1}\right) \backslash\left(S \cup X^{\prime}\right)$. Then

$$
|A|,|B| \geq \delta(D)-\left|S \cup X^{\prime}\right| \geq n / 2-k_{0} \text {. }
$$

Moreover, $A \cap B=\emptyset$ as otherwise we could extend our $(S, J, T)$-system $\left(\mathcal{P}_{j}\right)_{j \in J}$ by adding the path $P_{k}:=s_{k} x s_{1}$ where $x \in A \cap B$, a contradiction to the choice of $\left(\mathcal{P}_{j}\right)_{j \in J}$. In particular, this shows that the set $X^{\prime \prime}$ of all vertices outside $A \cup B \cup$ $S \cup X^{\prime}$ has size at most $2 k_{0}$ and thus, setting $Y:=S \cup X^{\prime} \cup X^{\prime \prime}$, we have that

$$
|Y| \leq 3 k_{0} \text {. }
$$


Note that $D$ does not contain an edge $\overrightarrow{a b}$ with $a \in A$ and $b \in B$. Indeed, otherwise we could extend $\left(\mathcal{P}_{j}\right)_{j \in J}$ by adding the path $P_{k}:=s_{k} a b s_{1}$. We will often use the following claim.

Claim 2. Let $a \in A$ and let $A^{\prime} \subseteq A$ be a set of size at least $k_{0}$. Then $N^{+}(a) \cap A^{\prime} \neq$ $\emptyset$. Similarly, if $b \in B$ and $B^{\prime} \subseteq B$ is a set of size at least $k_{0}$ then $N^{-}(b) \cap B^{\prime} \neq \emptyset$.

Suppose that $N^{+}(a) \cap A^{\prime}=\emptyset$. Then (11) together with the fact that $D$ does not contain an $A$ - $B$ edge implies that $d^{+}(a) \leq n-|B|-k_{0} \leq n / 2$, a contradiction. The proof of the second part of the claim is similar.

We say that a special vertex $s_{i}$ has out-type $A$ if $s_{i}$ sends at least $k_{0}$ edges to $A$. Similarly we define when $s_{i}$ has out-type $B$, in-type $A$ and in-type $B$. As $|Y|+2 k_{0} \leq$ $5 k_{0} \leq \delta(G)$, it follows that each $s_{i}$ has out-type $A$ or out-type $B$ (or both) and in-type $A$ or in-type $B$ (or both). Note that $s_{1}$ has in-type $B$ but not in-type $A$ whereas $s_{k}$ has out-type $A$ but not out-type $B$.

Claim 3. Let $j \in J$. If $s_{j}$ has out-type $A$ then $s_{j+1}$ has in-type $B$ but not in-type $A$. Similarly, if $s_{j}$ has out-type $B$ then $s_{j+1}$ has in-type $A$ but not in-type $B$.

Suppose that $s_{j}$ has out-type $A$ and $s_{j+1}$ has in-type $A$. Let $a \in N_{A}^{+}\left(s_{j}\right)$. Claim 2 implies that $a$ sends an edge to one of the at least $k_{0}$ vertices in $N_{A}^{-}\left(s_{j+1}\right)$. Let $a^{\prime} \in N_{A}^{-}\left(s_{j+1}\right)$ be such a neighbour of $a$. Then we could extend our $(S, J, T)$ system by adding the path $s_{j} a a^{\prime} s_{j+1}$, a contradiction. The proof of the second part of Claim 3 is similar.

Claim 4. No vertex in $B$ sends an edge to $A$.

Suppose that $\overrightarrow{b^{*} a^{*}}$ is an edge of $D$, where $a^{*} \in A$ and $b^{*} \in B$. Given vertices $a \in A$ and $b \in B$, put $N_{a b}:=N^{+}(a) \cap N^{-}(b)$. Note that $N_{a b} \subseteq Y$ and $a, b \notin$ $N^{+}(a) \cup N^{-}(b)$ as $D$ does not contain an $A$-B edge. Thus

$$
\left|N_{a b}\right| \geq 2 \delta(D)-(n-2)=\left(2\left\lceil\frac{n+k}{2}\right\rceil-2\right)-(n-2) \geq k .
$$

Let us now show that no special vertex $s_{i}$ with $i \in J$ has out-type $B$. So suppose $i \in J$ and $s_{i}$ has out-type $B$. Then Claim 3 implies that $s_{i+1}$ has in-type $A$. By Claim 2 some of the at least $k_{0}$ vertices in $N_{A}^{-}\left(s_{i+1}\right)$ receives an edge from $a^{*}$. Let $a^{\prime}$ be such a vertex. Similarly, some of the vertices in $N_{B}^{+}\left(s_{i}\right)$ sends an edge to $b^{*}$. Let $b^{\prime}$ be such a vertex. Then we could extend our $(S, J, T)$-system by adding the path $s_{i} b^{\prime} b^{*} a^{*} a^{\prime} s_{i+1}$, a contradiction. This shows that whenever $s_{i}$ is a special vertex of out-type $B$ then $i \notin J$. Let $Q$ denote the set of such vertices $s_{i}$. Note that $s_{k} \notin Q$ as $s_{k}$ does not have out-type $B$. Thus each special vertex in $Q$ forbids one index in $J$. Altogether this shows that

$$
|J| \leq k-1-|Q| .
$$

Let $S_{A}$ be the set of all those special vertices $s_{i}$ with $1 \leq N_{A}^{-}\left(s_{i}\right)<k_{0}$. Let $S_{B}$ be the set of all those special vertices $s_{i}$ with $1 \leq N_{B}^{+}\left(s_{i}\right)<k_{0}$. Let $A^{*}$ be the set of all those vertices in $A$ which do not send an edge to some vertex in $S_{A}$. Then $\left|A^{*}\right|>|A|-k k_{0}$. Similarly, let $B^{*}$ be the set of all those vertices in $B$ which do not receive an edge from some vertex in $S_{B}$. Then $\left|B^{*}\right|>|B|-k k_{0}$. 


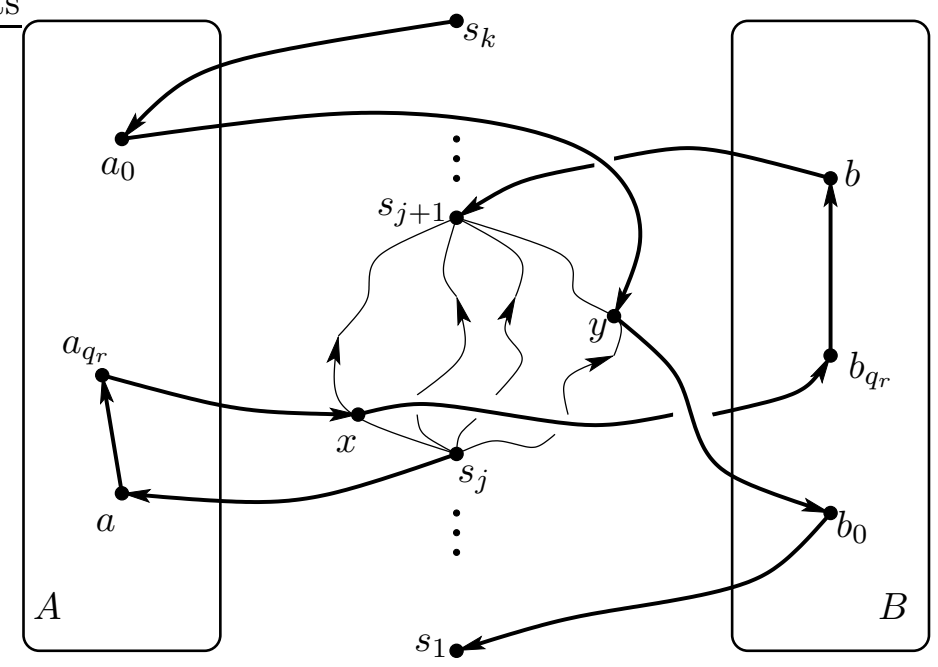

Figure 3. Modifying our $(S, J, T)$-system in the proof of Claim 4.

Consider any pair $a, b$ with $a \in A^{*}$ and $b \in B^{*}$. As each special vertex in $N_{a b}$ belongs to $Q$, it follows that

$$
\left|N_{a b} \backslash S\right| \geq\left|N_{a b}\right|-|Q| \stackrel{\sqrt[2]{\geq}}{\geq} k-|Q| \stackrel{\sqrt[3]{>}}{>}|J| .
$$

Suppose first that $J \neq \emptyset$. Given $j \in J$, let $X_{j}^{\prime \prime}$ be the union of $X^{\prime \prime}$ with the set of all vertices lying in the interior of paths in $\mathcal{P}_{j}$. As $N_{a b} \subseteq Y$ there must be an index $j_{a b} \in J$ such that $N_{a b}$ contains at least two vertices in $X_{j_{a b}}^{\prime \prime}$. Note that $\left|A^{*}\right|,\left|B^{*}\right|>2 k_{0} k$. Thus there are $2 k_{0}+1$ disjoint pairs $a, b$ for which this index $j_{a b}$ must be the same. Let $a_{q}, b_{q}\left(q=0, \ldots, 2 k_{0}\right)$ denote these pairs and let $j \in J$ denote the common index.

Note that $s_{j}$ has out-type $A$ since we have seen before that no special vertex $s_{i}$ with $i \in J$ has out-type $B$. Claim 3 now implies that $s_{j+1}$ has in-type $B$. Pick vertices $a \in N_{A}^{+}\left(s_{j}\right)$ and $b \in N_{B}^{-}\left(s_{j+1}\right)$ such that $a \neq a_{0}$ and $b \neq b_{0}$. Claim 2 implies that there are indices $q_{1}, \ldots, q_{k_{0}}$ such that $a$ sends an edge to each $a_{q_{r}}$. Apply Claim 2 again to find an index $r \leq k_{0}$ such that $b$ receives an edge from $b_{q_{r}}$. Let $x \in N_{a_{q_{r}} b_{q_{r}}}$ and $y \in N_{a_{0} b_{0}}$ be distinct vertices such that $x, y \in X_{j}^{\prime \prime}$. We can now modify our $(S, J, T)$-system to obtain an $\left(S, J \cup\{k\}, T^{\prime}\right)$-system in $D$ by replacing $\mathcal{P}_{j}$ with the single path $s_{j} a a_{q_{r}} x b_{q_{r}} b s_{j+1}$ and adding the $s_{k^{-}} s_{1}$ path $s_{k} a_{0} y b_{0} s_{1}$ (see Figure 3). If $J=\emptyset$ then we just add the $s_{k}-s_{1}$ path (which is still guaranteed by (44)). In both cases this contradicts the choice of our $(S, J, T)$-system and completes the proof of Claim 4 .

Claim 5. Let $a \in A$ and let $A^{\prime} \subseteq A$ be a set of size at least $k_{0}$. Then $N^{-}(a) \cap A^{\prime} \neq$ $\emptyset$. Similarly, if $b \in B$ and $B^{\prime} \subseteq B$ is a set of size at least $k_{0}$ then $N^{+}(b) \cap B^{\prime} \neq \emptyset$.

Using Claim 4, this can be shown similarly as Claim 2,

Let $S_{A}^{+}$be the set of all those special vertices which send an edge to $A$ and let $S_{A}^{-}$ be the set of all those special vertices which receive an edge from $A$. Define $S_{B}^{+}$ and $S_{B}^{-}$similarly. Note that these sets are not disjoint. The proof of the next claim 
is similar to that of Claim 3. (To prove the second and third part of Claim 6 we use Claim 5 instead of Claim 2.)

Claim 6. If $j \in J$ and $s_{j} \in S_{A}^{+}$then $s_{j+1}$ cannot have in-type $A$. If $j-1 \in J$ and $s_{j} \in S_{A}^{-}$then $s_{j-1}$ cannot have out-type $A$. If $j \in J$ and $s_{j} \in S_{B}^{+}$then $s_{j+1}$ cannot have in-type $B$. Finally, if $j-1 \in J$ and $s_{j} \in S_{B}^{-}$then $s_{j-1}$ cannot have out-type B.

Let $q_{A}^{+}:=\left|S_{A}^{+}\right|$and define $q_{A}^{-}, q_{B}^{+}$and $q_{B}^{-}$similarly. Let $\bar{Y}:=V(D) \backslash Y=A \cup B$ and $X:=X^{\prime} \cup X^{\prime \prime}=Y \backslash S$. Consider any pair $a, b$ with $a \in A$ and $b \in B$. Then

$$
\left\lceil\frac{n+k}{2}\right\rceil-1 \leq\left|N^{+}(a)\right| \leq q_{A}^{-}+\left|N_{X}^{+}(a)\right|+\left|N_{\bar{Y}}^{+}(a)\right|
$$

and

$$
\left\lceil\frac{n+k}{2}\right\rceil-1 \leq\left|N^{-}(b)\right| \leq q_{B}^{+}+\left|N_{X}^{-}(b)\right|+\left|N_{\bar{Y}}^{-}(b)\right| .
$$

Since $N_{\bar{Y}}^{+}(a) \cap N_{\bar{Y}}^{-}(b)=\emptyset$ (as $D$ does not contain an $A$ - $B$ edge) and $a, b \notin N_{\bar{Y}}^{+}(a) \cup$ $N_{\bar{Y}}^{-}(b)$ we have

$$
\left|N_{\bar{Y}}^{+}(a)\right|+\left|N_{\bar{Y}}^{-}(b)\right| \leq|\bar{Y}|-2=n-|X|-k-2 .
$$

Adding (5) and (6) together now gives

$$
2\left\lceil\frac{n+k}{2}\right\rceil-2 \leq q_{A}^{-}+q_{B}^{+}+\left|N_{X}^{+}(a)\right|+\left|N_{X}^{-}(b)\right|+n-|X|-k-2 .
$$

Hence

$$
\left|N_{X}^{+}(a) \cap N_{X}^{-}(b)\right| \geq\left|N_{X}^{+}(a)\right|+\left|N_{X}^{-}(b)\right|-|X| \geq 2\left\lceil\frac{n+k}{2}\right\rceil-n+k-q_{A}^{-}-q_{B}^{+} \geq 2 k-q_{A}^{-}-q_{B}^{+} .
$$

Similarly, using Claim 4, one can show that

$$
\left|N_{X}^{-}(a) \cap N_{X}^{+}(b)\right| \geq 2 k-q_{A}^{+}-q_{B}^{-} .
$$

Consider any $j \in J$. Recall that by Claim 3 we have that either $s_{j}$ has outtype $A$ and $s_{j+1}$ has in-type $B$ or $s_{j}$ has out-type $B$ and $s_{j+1}$ has in-type $A$. Let $J_{A B}$ denote the set of all those indices $j \in J$ for which the former holds and let $J_{B A}$ be the set of all those $j \in J$ for which the latter holds. Our next aim is to estimate $j_{A B}:=\left|J_{A B}\right|$ and $j_{B A}:=\left|J_{B A}\right|$. Note that Claim 6 implies that if $s_{j} \in S_{B}^{+}$then $j \notin J_{A B}$. As $s_{k} \notin S_{B}^{+}$and $k \notin J$, this shows that

$$
j_{A B} \leq k-1-\left|S_{B}^{+} \backslash\left\{s_{k}\right\}\right|=k-1-\left|S_{B}^{+}\right|=k-1-q_{B}^{+} .
$$

Also, if $s_{j} \in S_{A}^{-}$then $j-1 \notin J_{A B}$ by Claim [6. As $s_{1} \notin S_{A}^{-}$, this shows that

$$
j_{A B} \leq k-1-\left|S_{A}^{-} \backslash\left\{s_{1}\right\}\right|=k-1-\left|S_{A}^{-}\right|=k-1-q_{A}^{-} .
$$

Adding these two inequalites gives

$$
j_{A B} \leq k-1-\frac{q_{A}^{-}+q_{B}^{+}}{2} .
$$

In order to give an upper bound for $j_{B A}$, note that if $s_{j} \in S_{A}^{+}$then $j \notin J_{B A}$ by Claim 6. Thus

$$
j_{B A} \leq k-1-\left|S_{A}^{+} \backslash\left\{s_{k}\right\}\right| \leq k-q_{A}^{+} .
$$


Also, if $s_{j} \in S_{B}^{-}$then $j-1 \notin J_{B A}$ by Claim 6 , Thus

$$
j_{B A} \leq k-1-\left|S_{B}^{-} \backslash\left\{s_{1}\right\}\right| \leq k-q_{B}^{-} .
$$

Adding these two inequalites gives

$$
j_{B A} \leq k-\frac{q_{A}^{+}+q_{B}^{-}}{2} .
$$

Our next aim is to show that $D$ contains a $(S, J \cup\{k\})$-system. This will complete the proof of Theorem 2 since it contradicts the choice of our $(S, J, T)$-system. Pick distinct vertices $a_{0} \in A, a_{j} \in N_{A}^{+}\left(s_{j}\right)$ for all $j \in J_{A B}, a_{j}^{\prime} \in N_{A}^{-}\left(s_{j+1}\right)$ for all $j \in J_{B A}, b_{0} \in B, b_{j} \in N_{B}^{-}\left(s_{j+1}\right)$ for all $j \in J_{A B}$ and $b_{j}^{\prime} \in N_{B}^{+}\left(s_{j}\right)$ for all $j \in J_{B A}$. Choose a vertex $x_{0} \in N_{X}^{+}\left(a_{0}\right) \cap N_{X}^{-}\left(b_{0}\right)$ and link $s_{k}$ to $s_{1}$ by the path $Q_{k}:=s_{k} a_{0} x_{0} b_{0} s_{1}$. (This can be done since the right hand side of (7) is at least 2.) To find the other paths, we distinguish two cases.

Case 1. $j_{B A} \leq j_{A B}$

For all $j \in J_{B A}$ we pick a vertex $x_{j} \in N_{X}^{-}\left(a_{j}^{\prime}\right) \cap N_{X}^{+}\left(b_{j}^{\prime}\right)$ such that all these $x_{j}$ are pairwise distinct and distinct from $x_{0}$. Inequalities (8) and (10) together imply that this can be done. Inequality (7) together with the fact that

$$
2 k-q_{A}^{-}-q_{B}^{+}-1-j_{B A} \stackrel{(9)}{\geq} 2 j_{A B}+1-j_{B A} \geq j_{A B}+1,
$$

implies that for all $j \in J_{A B}$ we can now pick a vertex $x_{j} \in N_{X}^{+}\left(a_{j}\right) \cap N_{X}^{-}\left(b_{j}\right)$ such that $x_{0}$ and all the $x_{j}(j \in J)$ are pairwise distinct. If $j \in J_{A B}$ we link $s_{j}$ to $s_{j+1}$ by the path $Q_{j}:=s_{j} a_{j} x_{j} b_{j} s_{j+1}$. If $j \in J_{B A}$ we link $s_{j}$ to $s_{j+1}$ by the path $Q_{j}:=s_{j} b_{j}^{\prime} x_{j} a_{j}^{\prime} s_{j+1}$. The paths $Q_{j}(j \in J)$ and $Q_{k}$ are internally disjoint and have length 4 , so they form an $(S, J \cup\{k\})$-system, as required.

Case 2. $j_{B A}>j_{A B}$

We proceed similiarly as in Case 1 , but this time we choose the vertices $x_{j} \in$ $N_{X}^{+}\left(a_{j}\right) \cap N_{X}^{-}\left(b_{j}\right)$ for all $j \in J_{A B}$ first. As

$$
2 k-q_{A}^{+}-q_{B}^{-}-1-j_{A B} \stackrel{10}{\geq} 2 j_{B A}-1-j_{A B}>j_{B A}-1,
$$

inequality (8) implies that we can then pick the vertices $x_{j} \in N_{X}^{-}\left(a_{j}^{\prime}\right) \cap N_{X}^{+}\left(b_{j}^{\prime}\right)$ for all $j \in J_{B A}$. The paths $Q_{j}(j \in J)$ and $Q_{k}$ are then defined as before. This completes the proof of Theorem 2 ,

Note that throughout the proof, the paths we constructed always had length at most 6 (the only case where they had length exactly 6 was in the proof of Claim 44). This means that the proof can easily be translated into polynomial algorithm so that the exponent of the running time does not depend on $k$ : We simply start with any $(S, J, T)$-system with $J \subseteq I$. Now we go through the steps of the proof and find a 'better' $\left(S, J^{\prime}, T^{\prime}\right)$-system with $J^{\prime} \subseteq I$. Claim 1 implies that for fixed $k$ we only need to do this a bounded number of times. Since the paths we need have length at most 6 and there are only a bounded number of cases to consider in the proof, it is clear that one can find the better system in polynomial time with exponent independent of $k$. Altogether this means that the problem of finding a cycle encountering a given sequence of $k$ vertices is fixed parameter tractable for digraphs whose minimum degree satisfies the conditon in Theorem 2 (where $k$ is 
the fixed parameter). The same applies to the problem of linking $\ell$ given pairs of vertices. In general, even the problem of deciding whether a digraph is 2-linked is already NP-complete [10]. For a survey on fixed parameter tractable digraph problems, see [8].

\section{ACKNOWLEDGEMENT}

We are grateful to Andrew Young for reading through the manuscript.

\section{REFERENCES}

[1] J. Bang-Jensen and G. Gutin, Digraphs: Theory, Algorithms and Applications, Springer, 2000 .

[2] B. Bollobás and A. Thomason, Highly linked graphs, Combinatorica 16 (1996), 313-320.

[3] G. Chen, R.J. Faudree, R.J. Gould, L. Lesniak and M.S. Jacobson, Linear forests and ordered cycles, Discussiones Mathematicae - Graph theory 24 (2004), 47-54.

[4] Y. Egawa, R. Faudree, E. Györi, Y. Ishigami, R. Schelp and H. Wang, Vertex-disjoint cycles containing specified edges, Graphs and Combinatorics 16 (2000), 81-92.

[5] M. Ferrara, R. Gould, G. Tansey and T. Whalen, On H-linked graphs, Graphs and Combinatorics 22 (2006), 217-224.

[6] A. Ghouila-Houri, Une condition suffisante d'existence d'un circuit Hamiltonien, C. R. Acad. Sci. Paris 251 (1960), 495-497.

[7] R. Gould, A.V. Kostochka and G. Yu, On minimum degree implying that a graph is $H$-linked, SIAM J. on Discrete Math., to appear.

[8] G. Gutin and A. Yeo, Some parameterized problems on digraphs, preprint 2007.

[9] M.C. Heydemann and D. Sotteau, About some cyclic properties in digraphs, J. Combinatorial Theory B 38 (1985), 261-278.

[10] S. Fortune, J.E. Hopcroft, J. Wyllie, The directed subgraph homeomorphism problem, Theoretical Computer Science 10 (1980), 111-121.

[11] H.A. Jung, Eine Verallgemeinerung des $k$-fachen Zusammenhangs für Graphen, Math. Annalen 187 (1970), 95-103.

[12] K. Kawarabayashi, A. Kostochka and G. Yu, On sufficient degree conditions for a graph to be $k$-linked, Combinatorics, Probability and Computing, to appear.

[13] H. Kierstead, G. Sarközy and S. Selkow, On k-ordered Hamiltonian graphs, J. Graph Theory 32 (1999), 17-25.

[14] A. Kostochka and G. Yu, An extremal problem for $H$-linked graphs, J. Graph Theory 50 (2005), 321-339.

[15] A. Kostochka and G. Yu, Minimum degree conditions for $H$-linked graphs, Discrete Applied Math., to appear.

[16] Y. Manoussakis, $k$-linked and $k$-cyclic digraphs, J. Combinatorial Theory B 48 (1990), 216226.

[17] R. Thomas and P. Wollan, An improved extremal function for graph linkages, European Journal of Combinatorics 26 (2005), 309-324.

[18] C. Thomassen, Note on highly connected non-2-linked digraphs, Combinatorica 11 (1991), 393-395.

Daniela Kühn, Deryk Osthus

School of Mathematics

University of Birmingham

Edgbaston

Birmingham

B15 2TT

UK

E-mail addresses: $\{$ kuehn,osthus $\}$ maths.bham.ac.uk 